\section{Myelination gets direct}

\section{By Lauren Martz, Staff Writer}

Separate groups at the Case Western Reserve University School of Medicine and the Stanford University School of Medicine have developed similar approaches to directly reprogram rodent fibroblasts into oligodendrocyte progenitor cells. ${ }^{1,2}$ The direct lineage conversion method could be a safe and fast way to supply cells for myelination disorder cell therapies. Now, the teams need to show that their methods also reprogram human fibroblasts.

Myelination disorders include leukodystrophies and autoimmune conditions such as multiple sclerosis (MS). In all cases, loss of the myelin sheaths around axons of neurons impairs nerve firing and causes nervous system deficiencies.

Most available treatments for myelination disorders aim to slow demyelination or treat symptoms, but none repair or replace myelin.

One starting point for that goal is oligodendrocyte progenitor cells (OPCs), which are components of the CNS white matter that generate oligodendrocytes, the cells that produce myelin during development and after injury in the CNS.

But current sources of OPCs are limited to donor and embryonic stem cell tissues, both of which carry risks of immune rejection by the recipient. Donor OPCs also are in short supply, and cells derived from human embryonic stem cells come with a host of ethical issues and concerns about potential teratogenicity.

As an alternative, several groups including a team at Case Western have derived OPCs from rodent fibroblast-derived induced pluripotent stem (iPS) cells. ${ }^{3}$ iPS cells would be patient specific but require multiple manipulation steps, and the strategy has not yet yielded human OPCs.

The most recent approach for cell differentiation is direct lineage conversion-reprogramming somatic cells into a desired cell typewhich eliminates many manipulation steps.

The Stanford team is among the groups that have successfully reprogrammed rodent fibroblasts into neurons and neural stem cells. ${ }^{4}$

Now, the Case Western and Stanford teams have accomplished direct lineage conversion of mouse fibroblasts into OPCs. In papers published in the same issue of Nature Biotechnology, the teams used forced expression of OPC-specific transcription factors to reprogram rodent fibroblasts into OPCs that generated functional, myelin-producing oligodendrocytes.

The Case Western team was led by Paul Tesar, assistant professor of genetics and genome sciences. His group transfected mouse embryonic fibroblasts with a pool of lentiviral vectors carrying eight doxycyclineinducible transcription factors that were highly expressed in OPCs and known to play roles in oligodendrocyte development.

In culture, fibroblasts were reprogrammed with the transcription factor pools into cells that expressed OPC lineage genes and suppressed fibroblast-related genes. The cells differentiated into myelinating oligodendrocytes.

In cultured brain slices from early postnatal mice with hypomyelination due to loss of myelin basic protein ( $\mathrm{Mbp}$ ), transplantation of the reprogrammed OPCs with doxycycline led to myelination of the axons.

Transplantation of the reprogrammed cells into the dorsal spinal column of the adult mice with hypomyelination also generated Mbp-expressing myelin, suggesting the reprogrammed cells become functional oligodendrocytes in vivo.

The team then screened different combinations from the eight transcription factors and whittled the list of those required for reprogramming to three-SRY-box containing gene 10 (Sox10), oligodendrocyte transcription factor 2 (Olig2) and NK6 homeobox 2 (Nkx6-2). University of Bonn

The three transcription factors
of the cultured mouse fibroblasts into cells reprogrammed about $20 \%$ of the cultured mouse fibroblasts into cells
with OPC properties. The OPCs could be expanded in culture for at least five passages, and the set of three transcription factors also was sufficient to reprogram mouse lung fibroblasts into OPCs.

In the other paper, Marius Wernig and colleagues at Stanford used a group of transcription factors that partially overlapped with those used by the Case Western group to reprogram both mouse and rat fibroblasts into OPCs.

Wernig is assistant professor of pathology at Stanford's Institute for Stem Cell Biology and Regenerative Medicine.

The Stanford team first used a pool of lentiviruses carrying 10 oligodendrocyte-specific transgenes to reprogram mouse embryonic fibroblasts into OPCs. The team then shrank the cocktail to three transgenes-Sox10, Olig2 and zinc finger protein 536 (Zfp536)-sufficient to induce effective reprogramming.

The set of three transcription factors reprogrammed rat fibroblasts with about $15 \%$ purity. The OPCs differentiated into both oligodendrocytes and astrocytes in culture.

In dorsal root ganglion neurons with extended axon beds, coculture with the reprogrammed OPCs gave rise to Mbp-expressing cells, suggesting the OPCs effectively myelinated the axons.

Wernig and colleagues used the same mouse model as the Case Western group but directly injected the reprogrammed rat cells into 
the corpus callosum and cerebellum brain sections of neonatal mice. Administration of doxycycline in drinking water induced sustained transgene expression and led to the formation of Mbp-expressing myelin around neurofilaments.

"Our next steps are to utilize this technology on human cells to produce patient-specific, functional oligodendrocyte progenitor cells and oligodendrocytes for use in understanding and treating human disorders of myelin," Tesar said.

Wernig's team also plans to look into ways to convert human fibroblasts into induced OPCs.

Mike Gresser, CSO of the Myelin Repair Foundation, said, "These human OPCs will be critical for in vitro studies of myelination and to evaluate myelin repair drug candidates in the human brain."

The Myelin Repair Foundation funded the work at Case Western.

Frank Edenhofer, head of stem cell engineering at the University of Bonn's Institute of Reconstructive Neurobiology, told SciBX, “The most critical point for the clinical realization of the approach is the adaptation to the human system. I expect this to be doable; however, it might need a different combination of transcription factors or other stimuli such as microRNA."

\section{Cell source alternatives}

An open question is whether the direct lineage conversion method will be better than available donor or stem cell sources.

"The key question for this approach is: What is the advantage of creating oligodendrocytes in this way specifically?" said Sheng Ding, professor of pharmaceutical chemistry at the University of California, San Francisco and a senior investigator at the Gladstone Institutes. "This is key because there are already other methods to create oligodendrocytes. We need to determine whether this would be the most practical, safe and useful method to make large masses of oligodendrocyte progenitors in vitro for clinical use."

Malin Parmar, associate professor of developmental neurobiology at Lund University, thinks direct conversion could indeed be the optimal approach. "The advantage of direct conversion is that you bypass the pluripotent stage and thus avoid the risk of tumor formation or overgrowth due to uncontrolled proliferation after transplantation. Another advantage is that it is generally quicker and easier, which also means cheaper" than other approaches, she said. "Quicker is good because the less time cells are kept in vitro, the fewer things can go wrong and the fewer steps needed for quality control."

Ding said bypassing the stem cell step does reduce the level of risk of tumorigenesis but added that "there is always a risk when transforming cells. The transformation can cause them to become tumorigenic or unstable, and you can introduce different dangers through gene modification. This still uses a gene integration method and carries the associated dangers" such as cancer or unexpected toxicities.

Both Ding and Edenhofer were concerned about the efficiency of the direct reprogramming methods.

"Based on the data reported, the conversion appears not to result in a homogeneous induced oligodendrocyte progenitor population. Instead, reprogrammed induced oligodendrocyte progenitor populations might represent a quite heterogeneous population of fully and partially reprogrammed cells," said Edenhofer.

Ding added, "The fact that the genetic reprogramming only reprograms a small percentage of cells to the target cell type is a problem because it may not generate a functional and useful population, and the contamination with cells that are not fully reprogrammed could be dangerous."

Wernig told SciBX that his team is working to improve the reprogramming process to yield higher numbers of OPCs.

\section{Therapeutic remyelination}

Regardless of where the oligodendrocytes come from, the therapeutic effect in specific human diseases remains to be shown.

One concern, said Gresser, is that "inadequate quantities of oligodendrocyte progenitor cells in the brains of MS patients might not be what limits myelin repair of demyelinated lesions. It is possible that inadequate remyelination is due to factors present in or near the lesions that limit the ability of the oligodendrocyte progenitor cells that are there to proliferate and/or differentiate into myelination oligodendrocytes that properly myelinate demyelinated axons."

He added, "It should not be taken for granted that introducing human oligodendrocyte progenitor cells into the brain of an MS patient will by itself result in good myelin repair."

Tesar told SciBX that the Myelin Repair Foundation filed for a patent covering the direct cell fate conversion of somatic cells into OPCs and oligodendrocytes, which was assigned to Case Western. The IP is available for licensing.

Wernig said that two years ago, Stanford filed a patent application for the direct conversion of fibroblasts to neurons with a possibility to also induce oligodendrocytes using the same methods. He said that for undisclosed reasons, a patent was never issued.

Martz, L. SciBX 6(19); doi:10.1038/scibx.2013.456

Published online May 16, 2013

\section{REFERENCES}

1. Najm, F.J. et al. Nat. Biotechnol.; published online April 14, 2013; doi:10.1038/nbt.2561

Contact: Paul J. Tesar, Case Western Reserve University School of Medicine, Cleveland, Ohio

e-mail: paul.tesar@case.edu

2. Yang, N. et al. Nat. Biotechnol.; published online April 14, 2013; doi:10.1038/nbt.2564

Contact: Marius Wernig, Stanford University School of Medicine, Stanford, Calif.

e-mail: wernig@stanford.edu

3. Najm, F.J. et al. Nat. Methods 8, 957-962 (2011)

4. Vierbuchen, T. et al. Nature 463, 1035-1041 (2010)

\section{COMPANIES AND INSTITUTIONS MENTIONED}

Case Western Reserve University School of Medicine,

Cleveland, Ohio

Gladstone Institutes, San Francisco, Calif.

Lund University, Lund, Sweden

Myelin Repair Foundation, Saratoga, Calif.

Stanford University School of Medicine, Stanford, Calif.

University of Bonn, Bonn, Germany

University of California, San Francisco, Calif. 